\title{
Left Ventricular Shape and Motion Reconstruction through a Healthy Model for Characterizing Remodeling after Infarction
}

\author{
M. De Craene ${ }^{1}$, P. Piro ${ }^{1}$, N. Duchateau ${ }^{2}$, P. Allain ${ }^{1}$, and E. Saloux ${ }^{3}$ \\ 1 Philips Research Paris, France \\ 2 CREATIS, UMR5220, INSERM U 1206, Univ. Lyon 1, France \\ 3 Caen University Hospital, France
}

\begin{abstract}
We introduce a framework for the statistical characterization of heart remodeling from both shape and dynamics of the left ventricle. Shape was characterized by thickness and radius maps, unfolded in a two-dimensional dense Bull's eye. Motion was represented as a mixture of affine transformations in an anatomical space of coordinates. Using this representation, a population can be projected (after defining spatiotemporal correspondences) to an atlas space built for a given reference population - here, healthy subjects using a classic PCA approach yielding a joint model of healthy shape and motion statistics. The reconstruction error on shape and motion can then be exploited to quantify remodeling abnormalities. We demonstrate these concepts on 48 healthy subjects and 62 patients with infarct ( 29 with one year follow-up) imaged with 3D echocardiography, analyzing a total of 139 sequences.
\end{abstract}

Keywords: Cardiac $\cdot$ Shape $\cdot$ Motion · Abnormality

\section{Introduction}

Cardiac remodeling can be defined as the capacity of the cardiac muscle to adapt its structure, shape and dynamics to adversarial stresses. Statistical atlases can describe the variability of normal and pathological cardiac shape and motion. Therefore, they appear as a potential way of quantifying cardiac remodeling in a population. If statistical cardiac shape models are commonly used for segmenting images in clinical practice [2], motion atlases have been mostly used as a research tool for comparing or stratifying populations $[1,4,5]$. The following two issues actually condition their translation to clinical use.

How to compute a distance to normality? Computing a statistical distance between one subject and a control group made of healthy individuals, previously aligned to the same reference, can highlight suspicious regions. Such a concept was often applied in neurologic images for revealing subtle volume changes. For cardiac imaging, it was applied to represent abnormalities of myocardial velocities [1] and shapes [10] as $p$-values.

How to compute a distance between subjects based on several features? Depending on the pathology and its severity, shape and motion will be affected 
differently. A complete analysis therefore requires combining these features in a relevant manner. A straightforward way is to concatenate features in a single vector per subject. For example, in a recent shape modelling challenge [9] aiming at classifying infarcted subjects, some authors combined end-systolic and enddiastolic shapes, thicknesses or radial displacements using PCA. An alternative when corroborating similar information for various modalities is to look for a common lower dimensional space where both information sources are projected. This unified lower-dimensional space can be used to reconstruct either inputs (for example in case of missing information) [6]. Finally, an alternative way of integrating heterogeneous features is to weigh their relative importance, as recently demonstrated with MKL, a non-linear dimensionality reduction technique that has been exploited to reveal relevant groups of subjects [7].

In this paper, we propose to exploit the reconstruction of cardiac shape and motion from low-dimensional coordinates to express both shape and motion abnormalities against a reference population of healthy subjects. We explicitly generate abnormality maps that quantify cardiac remodeling in a practical and intuitive way.

\section{Methods}

Patient population and data acquisition This paper combines data from two research protocols, approved by the ethical committee of the hospital (DIRECT and Reve II). Both protocols were conducted at the same institution and involved the same imaging team. Enrolled patients all gave informed consent. In total, 48 control subjects and 62 patients with infarcts were included. Table 1 summarizes characteristics and inclusion criteria of all populations.

Table 1. Patient characteristics for control and infarct populations.

\begin{tabular}{|l|l|l|l|}
\hline & Controls & REVE II & \multicolumn{2}{|l|}{$\begin{array}{l}\text { Infarcts } \\
\text { DIRECT }\end{array}$} \\
\hline $\mathbf{n}$ & $\mathbf{4 8}$ & $\mathbf{4 2}$ & $\mathbf{2 0}$ \\
\hline $\begin{array}{l}\text { Type of } \\
\text { infarct }\end{array}$ & Anterior & $t=0,12$ months \\
\hline $\begin{array}{l}\text { Time } \\
\text { points }\end{array}$ & $t=0$ & $t=0$ & $\begin{array}{l}\text { Anterior / Inferior } \\
\text { criteria }\end{array}$ \\
\hline $\begin{array}{l}\text { Inclusion } \\
\text { cardiovascu- } \\
\text { lar disease }\end{array}$ & $\begin{array}{l}\text { sessed by either scintig- } \\
\text { raphy or cardiac MRI. }\end{array}$ & $\begin{array}{l}\text { Acute infarct assessed by } \\
\text { STEM on ECG. Echo at } \\
\text { most 5 days after infarct. } \\
E F<\text { 50\%. }>\text { aki- } \\
\text { netic ant. AHA segments } \\
\text { on echo. }\end{array}$ \\
\hline
\end{tabular}

Shape representation Left Ventricular (LV) meshes were obtained from the Philips Qlab (3DQA) software. All meshes were resampled as described in [11]. The endocardium and the epicardium were represented by two distance maps storing 
the distance to the long axis. Different samplings were used at different mesh locations: spherical for the top and bottom and cylindrical for the middle parts. Averaging the distance maps of the endocardial and epicardial surfaces gave a representation of the mid-LV surface. We defined this map as the LV radius map. The difference of the two distance maps was defined as the thickness map. Both maps can be visualized as Bull's eye plots (see Fig. 1).

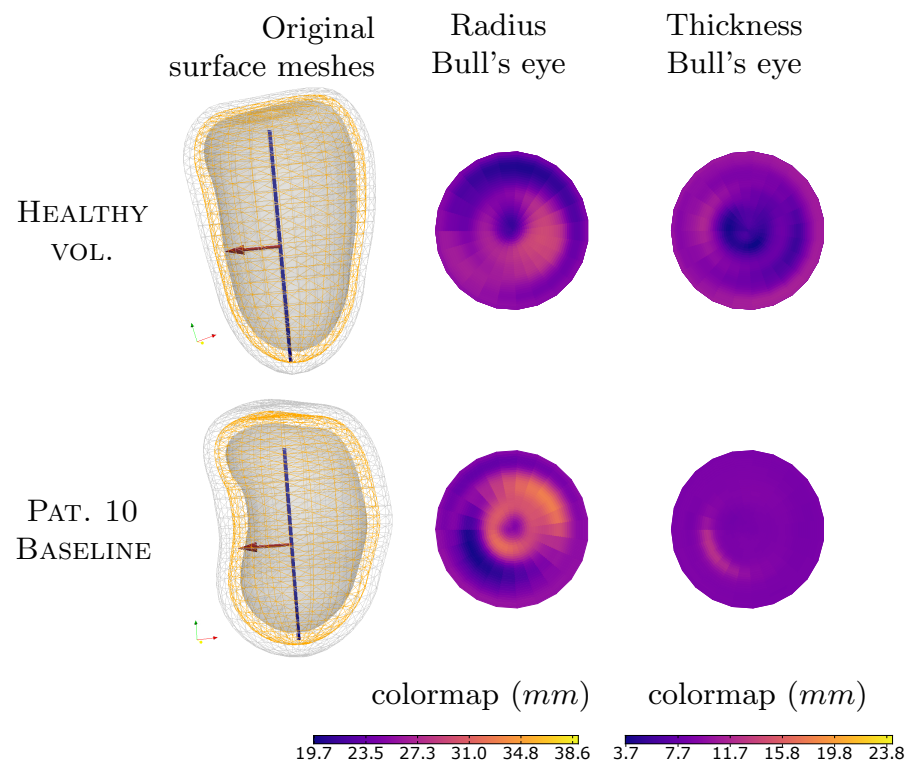

Fig. 1. Bull's eye representation of radius (center) and thickness maps (right) for one healthy volunteer and one infarcted patient (baseline).

Motion model We chose to represent cardiac motion by a poly-affine transformation model [4]. For this, we projected a dense non-rigid displacement field as obtained by a fast implementation of the Sparse Demons algorithm [8] on the regularization model from [11]. This regularization consists of three steps. First, a volumetric hexahedral mesh is built by connecting the endocardial, epicardial and mid-layers. Second, an anatomical system of coordinates is computed by orthogonalizing mesh edges at each vertex to define the Radial, Circumferential and Longitudinal (R, C, L) directions. Third, by geodesic integration of these vectors on the mesh, we obtain three scalar fields $(r, c$, and $l$ ) giving the local anatomical coordinates. A smooth partition of the mesh is then obtained through the definition of window functions.

$$
\varphi^{w}(\mathbf{x})=\frac{g^{w}(\mathbf{x})}{\sum_{n} g^{n}(\mathbf{x})}
$$


where $g^{w}(\mathbf{x})=\frac{1}{2 \pi \sigma_{w}} \exp \left(-\frac{\xi\left(\mathbf{x}, \mathbf{x}_{w}\right)^{2}}{2 \sigma_{w}{ }^{2}}\right)$ and $\xi$ represents the geodesic distance on the mesh to the center of the $w^{\text {th }}$ window. We used $N_{w}=17$ windows corresponding to the AHA segments. In each window, we estimated deformation and translation parameters to approximate the frame to frame displacement field $\mathbf{v}[8]$ by a linear model expressed in anatomical coordinates

$$
\boldsymbol{\ell}^{w}(\mathbf{x})=\mathbf{A}^{w}(r(\mathbf{x}) c(\mathbf{x}) l(\mathbf{x}))^{T}+\mathbf{b}^{w} \quad \text { for } w \in\left[0, N_{w}-1\right] .
$$

Minimizing the following error for each window

$$
E_{w}=\sum_{\mathbf{x} \in \Omega} \varphi^{w}(\mathbf{x})\left\|\ell^{w}(\mathbf{x})-\mathbf{v}(\mathbf{x})\right\|^{2}
$$

is equivalent to solving a linear system in each direction $d \in\{r, c, l\}$ :

$$
\begin{array}{r}
\left(\begin{array}{cccc}
\sum_{\mathbf{x}} \varphi^{w}(\mathbf{x}) r^{2}(\mathbf{x}) & \sum_{\mathbf{x}} \varphi^{w}(\mathbf{x}) r(\mathbf{x}) l(\mathbf{x}) & \sum_{\mathbf{x}} \varphi^{w}(\mathbf{x}) r(\mathbf{x}) c(\mathbf{x}) & \sum_{\mathbf{x}} \varphi^{w}(\mathbf{x}) r(\mathbf{x}) \\
\sum_{\mathbf{x}} \varphi^{w}(\mathbf{x}) r(\mathbf{x}) l(\mathbf{x}) & \sum_{\mathbf{x}} \varphi^{w}(\mathbf{x}) l^{2}(\mathbf{x}) & \sum_{\mathbf{x}} \varphi^{w}(\mathbf{x}) l(\mathbf{x}) c(\mathbf{x}) & \sum_{\mathbf{x}} \varphi^{w}(\mathbf{x}) l(\mathbf{x}) \\
\sum_{\mathbf{x}} \varphi^{w}(\mathbf{x}) r(\mathbf{x}) c(\mathbf{x}) & \sum_{\mathbf{x}} \varphi^{w}(\mathbf{x}) l(\mathbf{x}) c(\mathbf{x}) & \sum_{\mathbf{x}} \varphi^{w}(\mathbf{x}) c^{2}(\mathbf{x}) & \sum_{\mathbf{x}} \varphi^{w}(\mathbf{x}) c(\mathbf{x}) \\
\sum_{\mathbf{x}} \varphi^{w}(\mathbf{x}) r(\mathbf{x}) & \sum_{\mathbf{x}} \varphi^{w}(\mathbf{x}) l(\mathbf{x}) & \sum_{\mathbf{x}} \varphi^{w}(\mathbf{x}) c(\mathbf{x}) & \sum_{\mathbf{x}} \varphi^{w}(\mathbf{x})
\end{array}\right)\left(\begin{array}{c}
A_{d r} \\
A_{d l} \\
A_{d c} \\
b_{d}
\end{array}\right) \\
=\left(\begin{array}{c}
\sum_{\mathbf{x}} \varphi^{w}(\mathbf{x})\left(\mathbf{v}(\mathbf{x}) \cdot \hat{\mathbf{e}}_{d}(\mathbf{x})\right) r(\mathbf{x}) \\
\sum_{\mathbf{x}} \varphi^{w}(\mathbf{x})\left(\mathbf{v}(\mathbf{x}) \cdot \hat{\mathbf{e}}_{d}(\mathbf{x})\right) l(\mathbf{x}) \\
\sum_{\mathbf{x}} \varphi^{w}(\mathbf{x})\left(\mathbf{v}(\mathbf{x}) \cdot \hat{\mathbf{e}}_{d}(\mathbf{x})\right) c(\mathbf{x}) \\
\sum_{\mathbf{x}} \varphi^{w}(\mathbf{x})\left(\mathbf{v}(\mathbf{x}) \cdot \hat{\mathbf{e}}_{d}(\mathbf{x})\right)
\end{array}\right)
\end{array}
$$

Linear transformations per window (Eqn. 2) can then be converted to Cartesian coordinates by using the RLC basis vectors and then merged through the window functions $\varphi^{w}(\mathbf{x})$ to deform the whole ventricle.

Multi-scale approach To obtain a multi-scale representation, we first averaged all per-window systems and solved for a global linear transformation (global level). We then averaged the systems of basal, mid and apical windows and fitted the residual from the global reconstructed field (level 2). We finally proceeded similarly for the finest level (level 3, 17 AHA segments).

Healthy shape model PCA was applied to the thickness and radius maps on the healthy population. Both thickness and radius maps were vectorized before PCA. Also, two normalization strategies were considered. The first was to normalize all input maps by their average value. The second normalized them by the cavity volume. Plotting the explained variance ratio revealed that the volume normalization led to a more compact representation than the average one. Volume normalization was therefore chosen in our experiments. The same explained variance curve were also used to set the number of PCA components used for reconstruction. A $90 \%$ threshold led to 5 components for radius and 7 for thickness. 
Healthy motion model All frame to frame linear regression results $\mathbf{A}^{w}$ and $\mathbf{b}^{w}$ were interpolated (and scaled) along time to a normalized heart cycle of 30 frames. We used mitral valve opening and the beginning/end of the cycle as temporal landmarks. For computing healthy motion modes of variations, we combined different concatenation strategies. First, the global transformations were vectorized as described in [4] and concatenated across all time points. Computing a PCA across all subjects encoded the temporal variability of global healthy motion. Then, for the next resolutions (levels 2 and 3), matrices were vectorized and concatenated across the different spatial regions (3 at level 2, 17 at level 3) for each frame separately. This set of PCAs thus encoded the spatial variability at each frame seen over healthy subjects. The number of components to be used for each PCA in the healthy motion model was selected with a $90 \%$ threshold at each level on the explained variance curves. For levels 2 and 3, we took the maximum number of components over all time points after applying the threshold. This gave 18 components for the global level, and 8/12 components (per frame) for the subsequent levels.

Reconstruction For a given new subject, both shape and motion can be projected onto the healthy PCA output space and reconstructed. Computing the difference in the input spaces between the original and the reconstructed scalar/vector fields will indicate pathological deviations from the healthy model. For shape, as we treat two static scalar fields (radius and thickness), we defined the difference between original and healthy-based reconstructed maps as absolute radius/thickness abnormality. For motion, we computed the relative error between the displacement encoded by the original and reconstructed mesh points. This was done for the normalized cardiac cycle at the myocardial mid-layer. We defined a relative motion abnormality as

$$
\mu_{d}(\mathbf{x})=(\mathbf{u}(\mathbf{x}, \mathbf{t})-\hat{\mathbf{u}}(\mathbf{x}, \mathbf{t})) \frac{\min (1, \alpha\|\hat{\mathbf{u}}(\mathbf{x}, \mathbf{t})\|}{\|\hat{\mathbf{u}}(\mathbf{x}, \mathbf{t})\|}
$$

where $\mathbf{u}(\mathbf{x}, \mathbf{t})$ is the displacement obtained by chaining the poly-affine estimate of Eqn. 2 over time and $\hat{\mathbf{u}}(\mathbf{x}, \mathbf{t})$ is its reconstructed counterpart (from the healthy model). In Eqn. 5, the numerator of the second factor neutralizes the denominator for small displacement values.

\section{Results}

Shape reconstruction Fig. 2 shows the same input shapes as in Fig. 1 with the reconstructed radius map using the PCA normality model (overlaid as wireframe). The first case (healthy) was left out of the PCA training set. For the pathological case, the septal bulge is smoothed out by the normality model, resulting in negative and positive reconstruction errors along the septum, both at baseline and follow-up. 


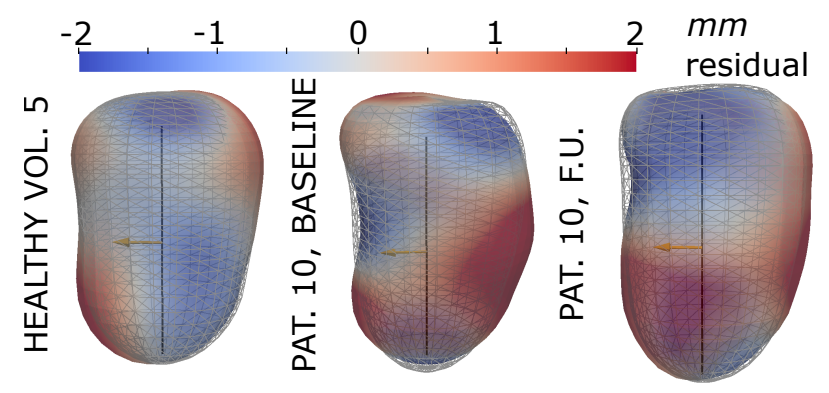

Fig. 2. Signed difference between the original radius map and its reconstruction (shown in wireframe) for a healthy subject and the patient shown in Fig. 1 (here at baseline and follow-up).

Motion reconstruction Fig. 3 (see links in the caption) plots the mid-layer meshes tracked around end-systolic frames at baseline and follow-ups (blue color). A difference in LV shape is visible between baseline and follow-up. The LV moved back to a more ellipsoidal shape at follow-up, which is known as reverse remodeling. The reconstructed healthy motion for this patient is plotted in green in Fig. 3. Differences between the healthy reconstruction and the patient's motion appear more pronounced at baseline than follow-up. This indicates that similarly to shape, motion abnormalities also reduced for this patient at follow-up. For taking a closer look at motion abnormalities, Fig. 4 plots the Bull's eye diagrams of $\mu_{d}$ (Eqn. 5) over time points close to the end of systole together with scar transmurality. The latter was segmented from late enhancement MR using the Segment (Medviso) software and resampled to the same Bull's eye as the motion abnormality maps. The $\mu_{d}$ maps return high abnormality values around the septal region, being reduced at follow-up. The extent and exact location of abnormalities differ between our index and the scar. This can first be explained by some potential misalignment when defining AHA segments in both modalities. Also, complex interaction mechanisms between stunned, infarcted and normal tissue (at baseline), or between normal, re-vascularized and scar tissue (at follow-up), can also explain the larger extent of the abnormality with our index.

Abnormality spreads Fig. 5a plots the $L^{2}$ norm of radius, thickness, motion and deformation abnormalities as box plots for controls and infarcts. Controls shape and motion abnormalities were computed by excluding that subject from the normality model database (leave-one-out approach). For each abnormality index, values were centered and normalized for the healthy population to have zero mean and unit standard deviation. Also, a two-sided Mann-Whitney rank test between controls and infarcts returned the $p$-values showed over the curly brackets . 
Joint analysis Finally, we selected one feature for shape and one for dynamics (radius and motion based on the $p$-values from Fig. 5a). These two indexes were rendered as a scatter plot in Fig. 5b. Similarly to Fig. 5a), both axes show the $L^{2}$ norm of the radius and $\mu_{d}$ abnormality maps. Controls, infarcts at baseline and infarcts at follow-up are plotted using different symbols. Most patients at follow-up had reduced relative motion abnormality $\mu_{d}$ compared to the overall distribution of baseline infarcts. A more detailed analysis would be required to compare the evolution in shape and motion abnormality values to the clinical outcome of each patient. Although the values in Fig. 5 do not seem to offer the same classification accuracy as some of the submissions to the shape modelling challenge [9], it should be emphasized that our results were extracted from a database of 3D US and not cine MR images. Also, as the patient populations differ, results can not be directly compared in terms of accuracy.

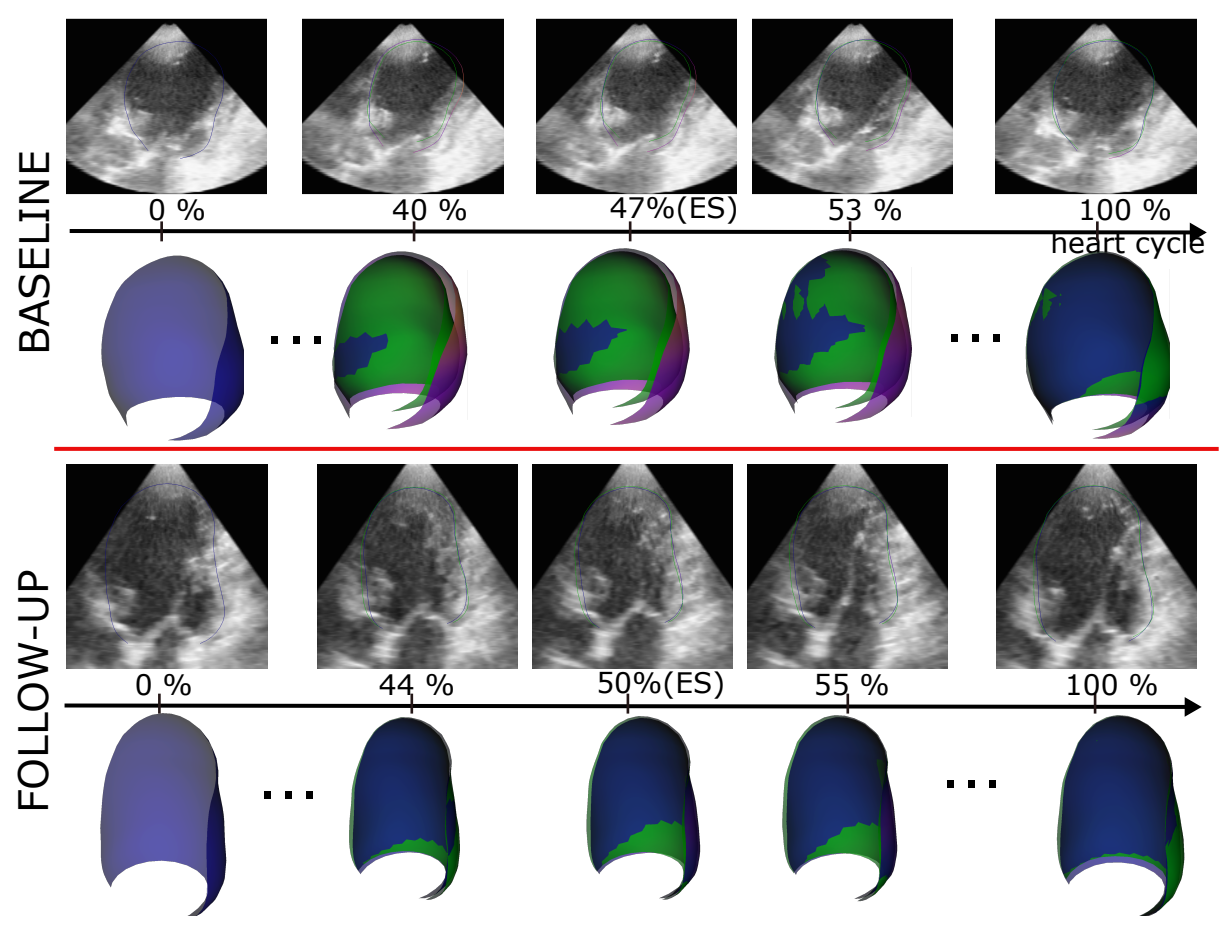

Fig. 3. LV meshes over the normalized cardiac cycle at baseline and follow-up for infarcted Patient \#4. The reconstruction based on the healthy model is shown in green. For an animated version, click on the following links: baseline, follow-up. 


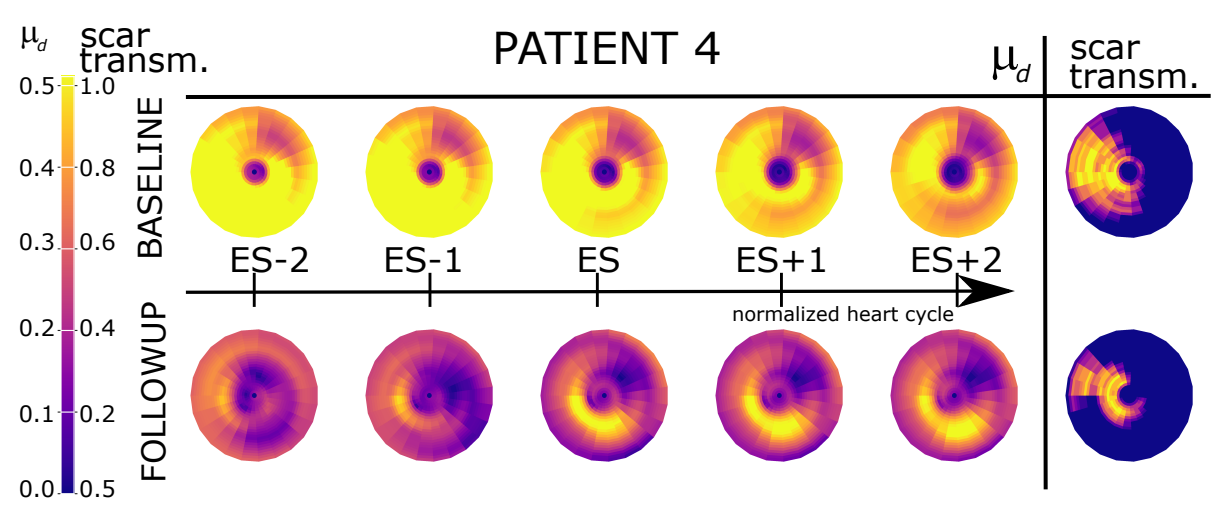

Fig. 4. Bull's eye plots of the motion $\left(\mu_{d}\right)$ abnormality computed over time for the same patient shown in Fig. 3 at baseline and follow-up. The scar transmurality (between 0 and 1) as measured from late enhancement images is plotted on the right.

a)

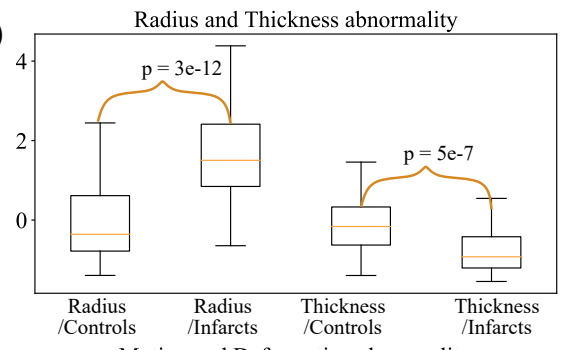

Motion and Deformation abnormality

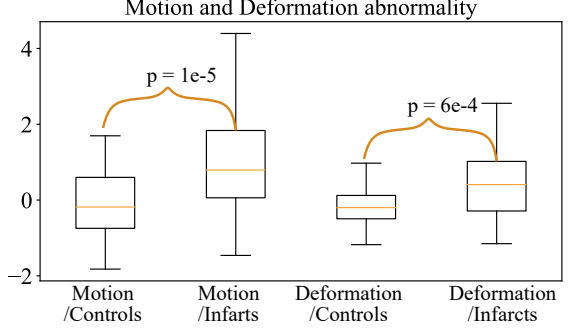

b)

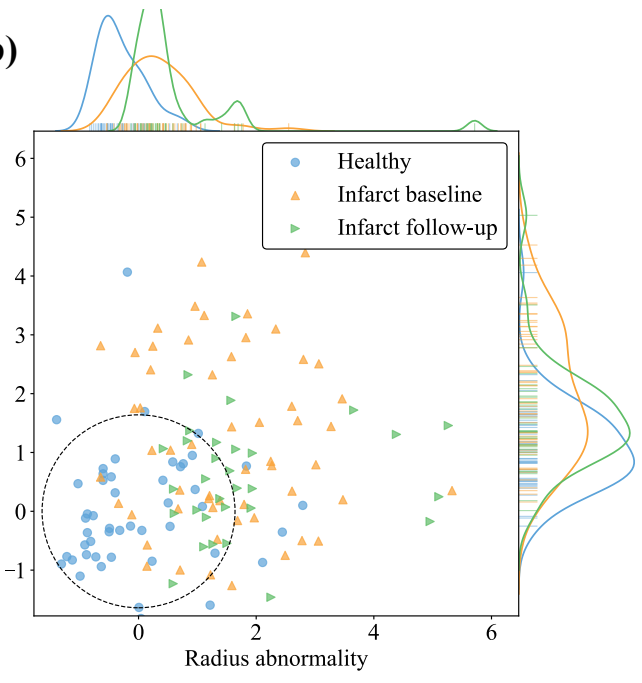

Fig. 5. a) Abnormality spreads for radius, thickness, motion and deformation among controls and infarcts. b) Distribution of the $L^{2}$ norm of $\mu_{d}$ and radius abnormality maps across healthy and infarcted populations, distinguishing between baseline and follow-up. 


\section{Conclusions}

We proposed a generic way to quantify abnormalities from different input descriptors, here illustrated on shape and motion to quantify cardiac remodeling in a population of patients with infarcts, comparing it to the normality pattern observed from a population of healthy subjects. A simple PCA approach was used to capture the healthy variability of both shape and motion descriptors. This model was then used on unseen cases to compute a reconstruction error that is used as an abnormality signature for both shape and motion. Including more pathological groups could emphasize different remodeling patterns. Also, the complex relationship between the presence of fibrosis (as revealed by late enhancement MR) and deformation requires to be further analyzed for a better comparison of scar and shape/motion abnormality maps.

\section{References}

1. Duchateau, N., et al.: A spatiotemporal statistical atlas of motion for the quantification of abnormal myocardial tissue velocities. Medical Image Analysis 15(3), 316-328 (2011).

2. Ecabert, O., et al.: Automatic model-based segmentation of the heart in CT images. IEEE Transactions on Medical Imaging 27(9), 1189-1201 (2008).

3. Fertin, M., et al.: Usefulness of serial assessment of b-type natriuretic peptide, troponin i, and c-reactive protein to predict left ventricular remodeling after acute myocardial infarction (from the REVE-2 Study). The American Journal of Cardiology 106(10), 1410-1416 (2010).

4. McLeod, K., et al.: Spatio-temporal tensor decomposition of a polyaffine motion model for a better analysis of pathological left ventricular dynamics. IEEE Transactions on Medical Imaging 34(7), 1562-1575 (2015).

5. Peressutti, D., Bai, W., Jackson, T., Sohal, M., Rinaldi, A., Rueckert, D., King, A.: Prospective identification of CRT super responders using a motion atlas and random projection ensemble learning. In: MICCAI, Part III. vol. 9351, pp. 493-500. Springer, LNCS (2015).

6. Puyol-Antón, E., et al.: A multimodal spatiotemporal cardiac motion atlas from mr and ultrasound data. Medical image analysis 40, 96-110 (2017)

7. Sanchez-Martinez, S., et al.: Characterization of myocardial motion patterns by unsupervised multiple kernel learning. Medical Image Analysis 35, 70-82 (2017).

8. Somphone, O., et al.: Fast myocardial motion and strain estimation in 3D cardiac ultrasound with sparse demons. In: 2013 IEEE 10th International Symposium on Biomedical Imaging. pp. 1182-1185 (2013).

9. Suinesiaputra, A., et al.: Statistical shape modeling of the left ventricle: myocardial infarct classification challenge. IEEE journal of biomedical and health informatics 22(2), 503-515 (2018).

10. Zhang, X., et al.: Atlas-based quantification of cardiac remodeling due to myocardial infarction. PloS one 9(10), e110243 (2014).

11. Zhou, Y., et al.: 3D harmonic phase tracking with anatomical regularization. Medical Image Analysis 26(1), 70-81 (2015). 